\title{
Article
}

\section{The Efficacy of S-1 as Adjuvant Chemotherapy for Resected Biliary Tract Carcinoma: A Propensity Score-Matching Analysis}

\author{
Yoichi Miyata ${ }^{1,2}, *\left(\mathbb{D}\right.$, Ryota Kogure ${ }^{3}$, Akiko Nakazawa ${ }^{2}$, Rihito Nagata ${ }^{4}$, Tetsuya Mitsui ${ }^{3}$, Riki Ninomiya ${ }^{3}$, \\ Masahiko Komagome ${ }^{3}$, Akira Maki ${ }^{3}$, Nobuaki Kawarabayashi ${ }^{5}$ and Yoshifumi Beck ${ }^{3}$ \\ 1 Department of Surgery, Asahi General Hospital, 1326 I, Asahi-shi, Chiba 289-2511, Japan \\ 2 Department of Surgery, National Defence Medical College, 3-2 Namiki, Tokorozawa-shi, \\ Saitama 359-8513, Japan; nakazawaa-sur@h.u-tokyo.ac.jp \\ 3 Department of Hepatobiliary Pancreatic Surgery, Saitama Medical Centre, Kamoda 1981, Kawagoe-shi, \\ Saitama 350-5500, Japan; ballershigh23and1@yahoo.co.jp (R.K.); tmitchiex@gmail.com (T.M.); \\ rickynino9222@gmail.com (R.N.); komagome@saitama-med.ac.jp (M.K.); akiramaki.md@gmail.com (A.M.); \\ ybeck@saitama-med.ac.jp (Y.B.) \\ 4 Hepato-Biliary-Pancreatic Surgery Division, Department of Surgery, Graduate School of Medicine, \\ The University of Tokyo, Bunkyo-ku, Tokyo 13-8655, Japan; NAGATAR-SUR@h.u-tokyo.ac.jp \\ 5 Department of Surgery, Gyoda General Hospital, Mochida 376, Gyoda-shi, Saitama 361-0056, Japan; \\ kawarabayashi@gyoda-hp.or.jp \\ * Correspondence: miyata-ham@umin.ac.jp
}

\section{check for} updates

Citation: Miyata, Y.; Kogure, R.; Nakazawa, A.; Nagata, R.; Mitsui, T.; Ninomiya, R.; Komagome, M.; Maki, A.; Kawarabayashi, N.; Beck, Y. The Efficacy of S-1 as Adjuvant Chemotherapy for Resected Biliary Tract Carcinoma: A Propensity Score-Matching Analysis. J. Clin. Med. 2021, 10, 925. https://doi.org/ $10.3390 /$ jcm10050925

Academic Editor: Saburo Matsubara

Received: 11 January 2021

Accepted: 19 February 2021

Published: 1 March 2021

Publisher's Note: MDPI stays neutral with regard to jurisdictional claims in published maps and institutional affiliations.

Copyright: (c) 2021 by the authors. Licensee MDPI, Basel, Switzerland. This article is an open access article distributed under the terms and conditions of the Creative Commons Attribution (CC BY) license (https:/ / creativecommons.org/licenses/by/ $4.0 /)$.

\begin{abstract}
Even though S-1 is a widely used chemotherapeutic agent, there is no evidence for its use in an adjuvant setting for biliary tract carcinoma (BTC). Patients who underwent surgical treatment for BTC between August 2007 and December 2018 were selected. Propensity score matching was performed between patients who received S-1 as adjuvant chemotherapy (S-1 group) and those who underwent surgical treatment alone (observation group). Of 170 eligible patients, 38 patients were selected in each group after propensity score matching. Among those in the matched cohort, both the median recurrence-free survival (RFS) and overall survival (OS) in the S-1 group were significantly longer than those in the observation group (RFS, 61.2 vs. 13.1 months, $p=0.033$; OS, not available vs. 28.2 months, $p=0.003$ ). A multivariate analysis of the OS revealed that perineural invasion and adjuvant S-1 chemotherapy were independent prognostic factors. According to a subgroup analysis of the OS, the S-1 group showed significantly better prognoses than the observation group among patients with perineural invasion $(p<0.001)$. S-1 adjuvant chemotherapy might improve the prognosis of BTC, especially in patients with perineural invasion.
\end{abstract}

Keywords: adjuvant chemotherapy; biliary tract carcinoma; propensity score matching; retrospective; S-1

\section{Introduction}

Biliary tract carcinoma (BTC) is a relatively rare cancer worldwide [1]. According to the World Health Organization classification, BTC includes perihilar and distal extrahepatic bile duct carcinoma and gallbladder carcinoma [2]. The surgical procedure for BTC depends on the location of the lesion. For example, major hepatectomy with extra bile duct resection is performed for perihilar carcinoma, and pancreaticoduodenectomy is the most common approach for distal cholangiocarcinoma. Even though radical resection is required to completely remove the tumour, the recurrence rate is reported to be high, around 50\% [3], and the overall survival (OS) rate remains poor.

The benefit of adjuvant chemotherapy after the surgical treatment of several advanced cancers, such as gastric cancer [4-6], colon cancer [7,8], and pancreatic cancer [9], is well established. Various adjuvant chemotherapy regimens are reported to improve the prognosis of patients with such cancers. However, in the case of BTC, the few large randomized trials on adjuvant chemotherapy conducted to date have produced unpromising results $[10,11]$ and the efficacy of adjuvant chemotherapy for BTC remains unknown. 
S-1 is an oral anti-cancer drug consisting of tegafur, 5-chloro-2,4-dihydroxypridine, and potassium oxonate [12,13]. The benefit of S-1 as an adjuvant chemotherapy has been reported for gastric cancer [4] and pancreatic cancer [9]. For BTC, several studies have shown that the efficacy of S-1 adjuvant chemotherapy varies [14,15], and its effectiveness remains debatable.

The aim of the present study was to retrospectively investigate the efficacy of S-1 administration as adjuvant chemotherapy after the surgical treatment of BTC. Because the patients with advanced carcinoma received adjuvant chemotherapy, we performed propensity score matching to reduce the inherent bias.

\section{Materials and Methods}

\subsection{Patient Selection}

Charts from two institutions were reviewed to select the patients who had undergone surgical treatment for BTC at Saitama Medical Centre and Gyoda General Hospital between August 2007 and December 2018. All the selected patients were pathologically diagnosed with BTC, including gallbladder carcinoma, perihilar cholangiocarcinoma, and distal cholangiocarcinoma. Patients who had received chemotherapies other than S-1 before and/or after their surgical treatment, had undergone $\mathrm{R} 2$ resection, or had not been able to receive S-1 adjuvant chemotherapy because they had died from postoperative complications within 90 days after surgery were excluded.

All the clinical, laboratory, radiologic, and pathological data were collected from electronic medical records. The study was conducted according to the guidelines of the Declaration of Helsinki and approved by the Institutional Review Board of Saitama Medical Centre, Saitama Medical University (No. 2002), and Gyoda General Hospital (No. 2019-1).

\subsection{Treatment Strategy and Follow Up}

The surgical treatment strategy was planned in accordance with the clinical status, such as pancreaticoduodenectomy for distal cholangiocarcinoma or major hepatectomy with extra bile duct resection for perihilar cholangiocarcinoma and gallbladder carcinoma. All the patients underwent adequate regional lymph node dissection, including the removal of hilar and pericholedochal nodes in the hepatoduodenal ligament, posterior and anterior pancreaticoduodenal nodes, and nodes along the common hepatic artery [16] Intraoperative pathological examination of the proximal and/or distal biliary tract margins was performed to confirm carcinoma-free margins using frozen tissue sections. If the biliary tract margin was positive for carcinoma, then additional biliary tract resection was performed until the margin was free (or to the maximum extent possible) from carcinoma.

Patients in each institution were followed up after their surgical treatment every 3 to 6 months, which consisted of basic blood examinations, including the carbohydrate antigen 19-9 (CA19-9) level, and imaging examinations were usually performed with contrastenhanced computed tomography. Additional imaging examinations were performed if recurrence was suspected. The end of the follow-up period was set as March 2019 or the date of death.

\subsection{Administration Criteria of Adjuvant S-1 Chemotherapy}

We considered the administration of S-1 (TS-1; Taiho, Tokyo, Japan) as adjuvant chemotherapy for patients to whom any of the following pathological findings applied: positive for lymphatic invasion and/or venous invasion and/or perineural invasion; positive for lymph node metastases, microscopic residual tumor, T status of T3 or T4. The patients also satisfied all of following criteria: Eastern Cooperative Oncology Group performance status (PS) of less than 2, adequate bone marrow function (leukocyte count $\geq 3000$ cells per cubic millimeter, hemoglobin concentration $\geq 8.0 \mathrm{~g} / \mathrm{dL}$, and platelet count $\geq 100,000$ cells per cubic millimeter), adequate liver function (total bilirubin concentration $\leq 2.0 \mathrm{mg} / \mathrm{dL}$, asparate aminotransferase concentration $\leq 100 \mathrm{IU} / \mathrm{L}$, and alanine aminotransferase concentration $\leq 100 \mathrm{IU} / \mathrm{L}$ ), adequate renal function (serum creatinine concentration $\leq 1.5 \mathrm{mg} / \mathrm{dL}$ ). 
These patients received oral S-1 twice daily at a dose matched to their body surface area (BSA) as follows: BSA $<1.25 \mathrm{~m}^{2}, 80 \mathrm{mg} /$ day; $1.25 \mathrm{~m}^{2} \leq \mathrm{BSA}<1.50 \mathrm{~m}^{2}, 100 \mathrm{mg} /$ day; and $1.50 \mathrm{~m}^{2} \leq \mathrm{BSA}, 120 \mathrm{mg} /$ day [13]. S-1 was administered for 28 days, followed by 14 days of rest in each 42-day cycle. Adjuvant S-1 chemotherapy was performed as long as possible unless the patients' condition were intolerable such as PS was higher than 2, or liver and/or renal disfunction. The patients who experienced recurrence were given adequate treatment, including the best supportive care.

\subsection{Statistical Analysis}

All the pathological diagnoses were recorded in accordance with the 8th edition of the Union for International Cancer Control TMN classification [16]. OS was defined as the interval from the date of surgical treatment to the date of death from any cause or the end of the follow-up period. Recurrence-free survival (RFS) was defined as the interval from the date of surgical treatment to the date of confirmed recurrence. The interval from the surgical treatment to the date of death or end of the follow-up period for patients without recurrence was also defined as RFS.

Continuous data were expressed as the median with range. Quantitative and categorized variables were compared using Wilcoxon's rank-sum test and the chi-squared test, respectively. RFS and OS were estimated using the Kaplan-Meier method, and differences in survival curves were compared using the log-rank test. The multivariate analysis of OS was performed using a Cox proportional-hazards model to the factors statistically significant on univariate analysis, and the results were expressed as the hazard ratio (HR) and $95 \%$ confidence interval $(95 \% \mathrm{CI})$.

Potential co-variables included in the propensity score matching were age, CA19-9 level, tumour (perihilar and distal extrahepatic bile duct carcinoma and gallbladder carcinoma), tumour differentiation, lymphatic invasion, venous invasion, perineural invasion, $\mathrm{T}$ status, $\mathrm{N}$ status, $\mathrm{R}$ status, and postoperative complications in accordance with the ClavienDindo classification [17]. Propensity scores were estimated using a logistic regression model, and the C-statistic for evaluating the goodness of fit was calculated. A one-to-one nearest-neighbour matching algorithm was applied with a calliper of 0.2.

$p$ values $\leq 0.050$ were considered statistically significant. All the statistical analyses were performed using JMP software (version 9.0.0; SAS Institute, Cary, NC, USA).

\section{Results}

\subsection{Patient Characteristics in the Entire Cohort}

During the study periods, 252 patients underwent surgical treatment for BTC. One patient who received chemotherapy before surgery, 23 who received adjuvant chemotherapies other than S-1, 24 treated with R2 resection, and 19 who died within 90 days after surgery without receiving S-1 adjuvant chemotherapy were excluded. Three patients who did not meet the administration criteria received adjuvant S- 1 chemotherapy and 12 patients who met the administration criteria received surgical treatment alone were also excluded. Finally, 170 patients were designated as the entire cohort (Figure 1).

The median age of the entire cohort was 74 (range, 42 to 90) years, and 106 (62\%) were male. There were $116(68 \%)$ cases of cholangiocarcinoma (49 (29\%) hilar cholangiocarcinoma and 67 [39\%] distal cholangiocarcinoma) and 54 (32\%) cases of gallbladder carcinoma. Hepatectomy was required in $51(30 \%)$ patients (35 (21\%) major hepatectomy and $16(9 \%)$ hepatopancreaticoduodenectomy) and 70 patients underwent pancreaticoduodenectomy. Lymphatic invasion, venous invasion, perineural invasion, and lymph node metastases were observed in $87(51 \%), 102(60 \%), 113(66 \%)$, and $71(42 \%)$ patients, respectively. R0 resection was achieved in $122(72 \%)$ patients. S-1 adjuvant chemotherapy was administered in $77(45 \%)$ patients, and the median duration of S-1 administration was 10.6 (range, 1.9 to 59.3) months. The profiles and tumour characteristics of the patients who received S-1 adjuvant chemotherapy (S-1 group) and surgical treatment alone (observation group) are shown in Table 1 . The median age of the S-1 group was significantly lower than that of 
the observation group $(p<0.001)$. Lymphatic invasion, venous invasion, and perineural invasion were observed significantly more often in the S-1 group than in the observation group ( $p=0.001,0.001$, and 0.005 , respectively). The proportion of patients with a T status of "T3 and T4" and N1 disease was also higher in the S-1 group ( $p<0.001$ for each). The R0 resection rate was comparable between two groups $(p=0.255)$.

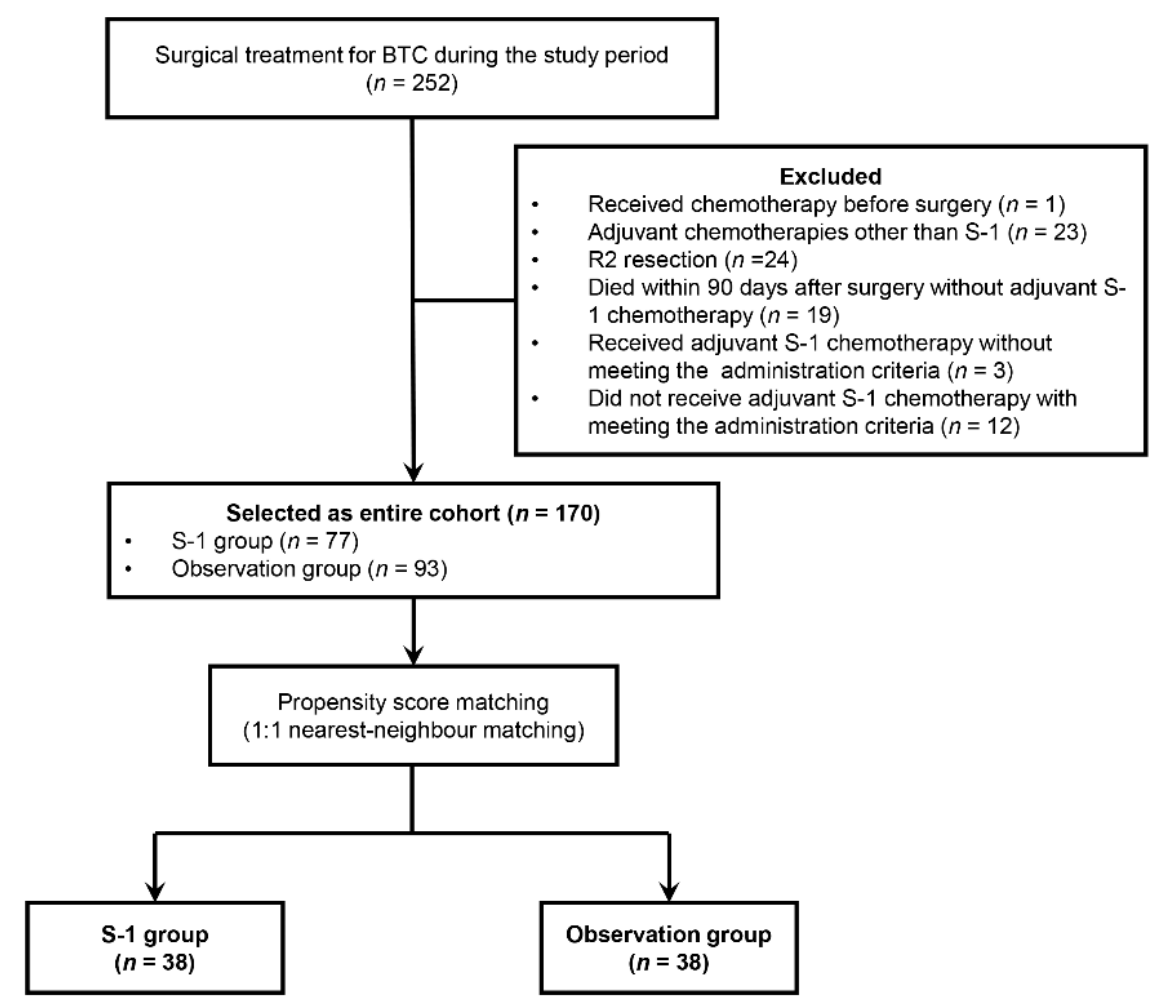

Figure 1. Flow chart of the patients included the study.

Table 1. Profiles and tumour characteristics of the patients in each group of the entire cohort.

\begin{tabular}{|c|c|c|c|}
\hline & $\begin{array}{c}\text { S-1 Group } \\
n=77\end{array}$ & $\begin{array}{l}\text { Observation Group } \\
\qquad n=93\end{array}$ & $p$ Value \\
\hline Age [y] & $70(44-87)$ & $75(42-90)$ & $<0.001 *$ \\
\hline Gender, male & $48(62)$ & $58(62)$ & 0.997 \\
\hline \multicolumn{4}{|l|}{ Diagnosis } \\
\hline Hilar cholangiocarcinoma & $20(26)$ & $29(31)$ & \\
\hline Distal cholangiocarcinoma & $39(51)$ & $28(30)$ & $0.018 *$ \\
\hline Gallbladder carcinoma & $18(23)$ & $36(39)$ & \\
\hline Serum CA19-9 [U/mL] & $91(1-33,564)$ & $53(1-2524)$ & 0.133 \\
\hline Hepatectomy & $23(30)$ & $28(30)$ & 0.973 \\
\hline Clavien-Dindo classification, III-V & $35(45)$ & $31(33)$ & 0.107 \\
\hline \multicolumn{4}{|l|}{ Pathological findings } \\
\hline Tumor differentiation, well & $25(32)$ & $37(40)$ & 0.230 \\
\hline Lymphatic invasion & $50(65)$ & $37(40)$ & $0.001 *$ \\
\hline Venous invasion & $57(74)$ & $45(48)$ & $0.001 *$ \\
\hline Perineural invasion & $61(79)$ & $52(56)$ & $0.005^{*}$ \\
\hline $\mathrm{T}$ status, $\mathrm{T} 3$ and $\mathrm{T} 4$ & $46(60)$ & $29(31)$ & $<0.001$ * \\
\hline N status, N1 & $48(62)$ & $23(25)$ & $<0.001$ * \\
\hline R status, R0 & $53(69)$ & $69(74)$ & 0.255 \\
\hline
\end{tabular}

* Statistical significance $(p<0.050)$. Values in parentheses are the percentages for categorical data or range for continuous data. CA19-9, carbohydrate antigen 19-9. 
The median RFS and OS of the entire cohort were 34.0 and 86.7 months, respectively. The median length of the follow-up interval was 50.6 months. Kaplan-Meier curves of the RFS and OS are shown in Figure S1.

\subsection{Patient Characteristics and Survival in the Matched Cohort}

After propensity score matching, 76 patients (38 in both the S- 1 and observation groups) were selected. The $\mathrm{C}$-statistic for the goodness of fit was 0.818 . Table 2 shows the profiles and tumour characteristics of the patients in each group in the matched cohort. Hilar cholangiocarcinoma, distal cholangiocarcinoma, and gallbladder carcinoma were present in $11(29 \%), 18(47 \%)$, and $9(24 \%)$ patients in the S-1 group, respectively, and in $10(26 \%), 17(45 \%)$, and $11(29 \%)$ patients in the observation group, respectively $(p=0.871)$.

Table 2. Profiles and tumour characteristics of the patients in each group of the matched cohort.

\begin{tabular}{cccc}
\hline & $\begin{array}{c}\text { S-1 Group } \\
n=38\end{array}$ & $\begin{array}{c}\text { Observation Group } \\
n=38\end{array}$ & $p$ Value \\
\hline Age [y] & $72(52-82)$ & $74(42-85)$ & 0.640 \\
Gender, male & $25(66)$ & $22(58)$ & 0.479 \\
Diagnosis & & & \\
Hilar cholangiocarcinoma & $11(29)$ & $10(26)$ & 0.871 \\
Distal cholangiocarcinoma & $18(47)$ & $17(45)$ & \\
Gallbladder carcinoma & $9(24)$ & $11(29)$ & 0.593 \\
Serum CA19-9 [U/mL] & $64(1-1807)$ & $68(1-2524)$ & 0.622 \\
Hepatectomy & $11(29)$ & $13(34)$ & 0.817 \\
Clavien-Dindo classification, III-V & $16(42)$ & $17(45)$ & \\
Pathological findings & & & 1.000 \\
Tumor differentiation, well & $14(37)$ & $14(37)$ & 0.634 \\
Lymphatic invasion & $25(66)$ & $23(61)$ & 0.807 \\
Venous invasion & $25(66)$ & $26(68)$ & 0.602 \\
Perineural invasion & $29(76)$ & $27(71)$ & 0.818 \\
T status, T3 and T4 & $20(53)$ & $21(55)$ & 0.817 \\
N status, N1 & $17(45)$ & $16(42)$ & 0.791 \\
R status, R0 & $29(76)$ & $28(74)$ &
\end{tabular}

Values in parentheses are the percentages for categorical data or range for continuous data. CA19-9, carbohydrate antigen 19-9.

The median interval from surgical treatment to the initiation of adjuvant S- 1 chemotherapy was 63 (range, 21 to 146) days, and the median duration of S-1 administration was 11.1 (range, 1.9 to 59.3) months.

The both median RFS and OS was significantly longer in the S-1 group than observation group (RFS, 61.2 vs. 13.1 months, $p=0.033$; OS, not available vs. 28.2 months, $p=0.003$ ) (Figure 2).

\subsection{Univariate and Multivariate Analysis of OS in the Matched Cohort}

The univariate and multivariate analysis of OS in the matched cohort is shown in Table 3. According to the univariate analysis, adjuvant S-1 chemotherapy as well as venous invasion and perineural invasion were significant predictors. The multivariate analysis revealed the presence of perineural invasion (Hazard ratio $[\mathrm{HR}]=6.038,95 \%$ CI, 1.709-29.153, $p=0.004$ ) without adjuvant S-1 chemotherapy ( $\mathrm{HR}=4.370,95 \% \mathrm{CI}$, $1.989-10.298, p<0.001$ ) was an independent poor prognostic factor. 


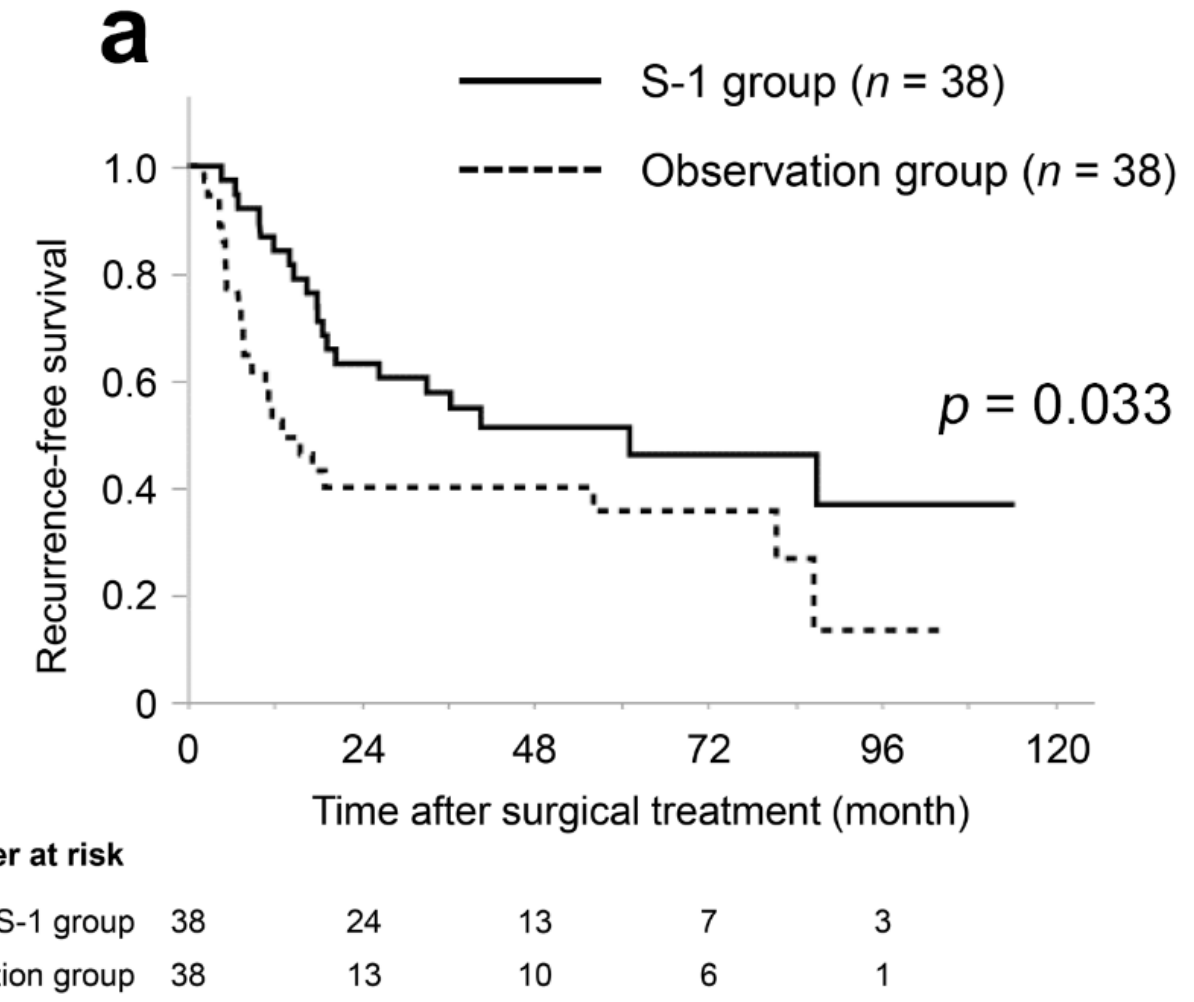

$\begin{array}{rrrrrr}\text { Number at risk } & & & & & \\ \text { S-1 group } & 38 & 24 & 13 & 7 & 3 \\ \text { Observation group } & 38 & 13 & 10 & 6 & 1\end{array}$

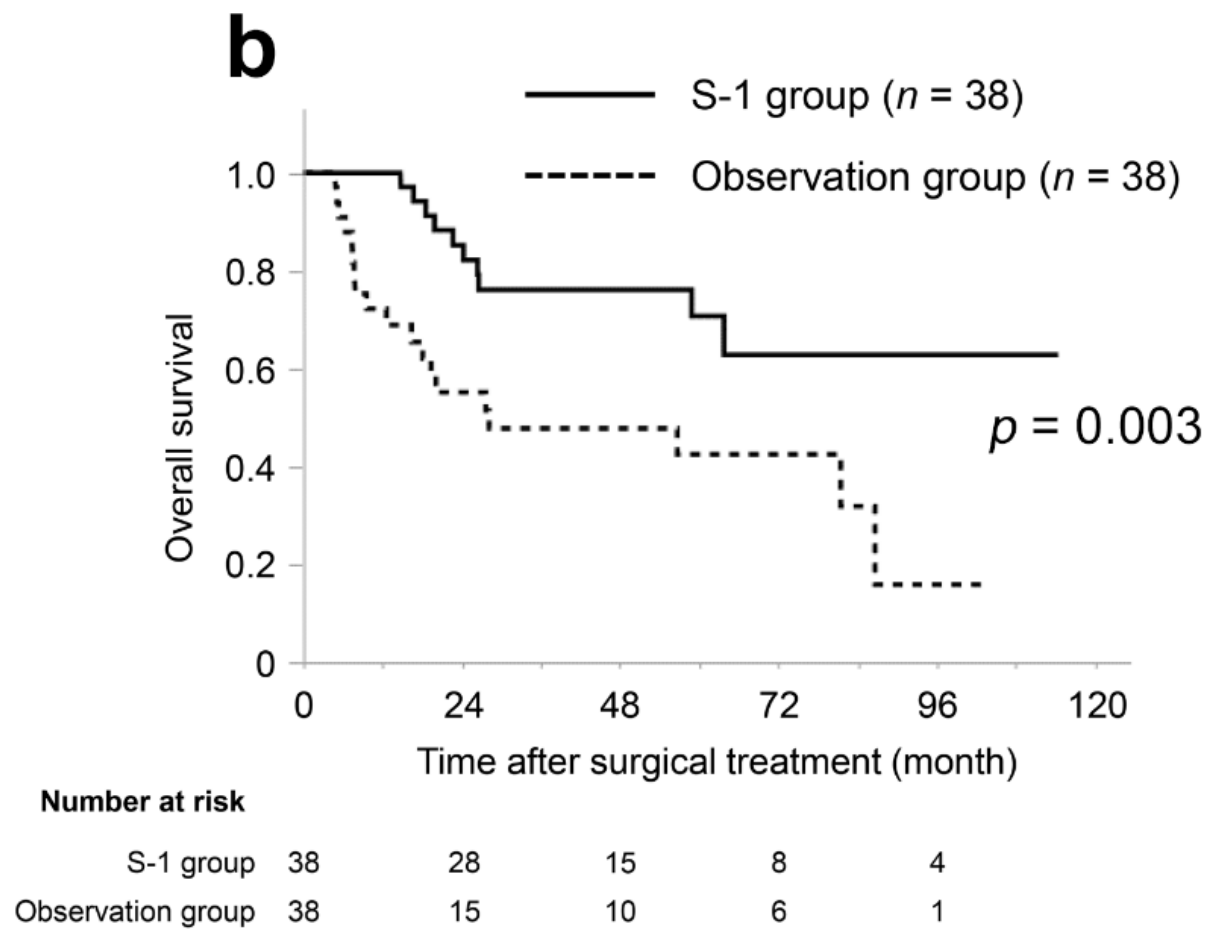

Figure 2. Kaplan-Meier curves of recurrence-free survival (a) and overall survival (b) in the matched cohort. The survival duration in the S-1 group was significantly longer than that in the observation group both in recurrence-free survival and overall survival ( $p=0.033$ and $p=0.003$, respectively). 
Table 3. Univariate and multivariate analysis of overall survival in the matched cohort.

\begin{tabular}{|c|c|c|c|c|c|c|c|}
\hline \multirow{2}{*}{ Variable } & & \multirow{2}{*}{$n$} & \multirow{2}{*}{$\begin{array}{l}\text { Median } \\
\text { (Months) }\end{array}$} & \multirow{2}{*}{$\frac{\text { Univariate }}{p \text { Value }^{\dagger}}$} & \multicolumn{3}{|c|}{ Multivariate } \\
\hline & & & & & HR & $95 \% \mathrm{CI}$ & $p$ Value $\ddagger$ \\
\hline \multirow[t]{2}{*}{ Age [y] } & $<65$ & 16 & 86.7 & \multirow{2}{*}{0.274} & & & \\
\hline & $\geq 65$ & 60 & 63.8 & & & & \\
\hline \multirow[t]{2}{*}{ Preoperative CA19-9 [U/mL] } & $<37$ & 23 & 86.7 & \multirow{2}{*}{0.317} & & & \\
\hline & $\geq 37$ & 53 & 58.9 & & & & \\
\hline \multirow[t]{2}{*}{ Clavien-Dindo classification } & $\mathrm{I}-\mathrm{II}$ & 43 & 86.7 & \multirow{2}{*}{0.666} & & & \\
\hline & III-V & 33 & 63.8 & & & & \\
\hline \multirow[t]{2}{*}{ Differentiation } & well & 28 & 86.7 & \multirow{2}{*}{0.134} & & & \\
\hline & not well & 48 & 58.9 & & & & \\
\hline \multirow[t]{2}{*}{ Lymphatic invasion } & no & 28 & 86.7 & \multirow{2}{*}{0.341} & & & \\
\hline & yes & 51 & 81.5 & & & & \\
\hline \multirow[t]{2}{*}{ Venous invasion } & no & 25 & 86.7 & \multirow{2}{*}{0.024 * } & & & \\
\hline & yes & 51 & 56.7 & & 1.342 & $0.510-4.102$ & 0.568 \\
\hline \multirow[t]{2}{*}{ Perineural invasion } & no & 20 & 86.7 & \multirow{2}{*}{$0.007 *$} & & & \\
\hline & yes & 56 & 56.7 & & 6.038 & $1.709-29.153$ & 0.004 * \\
\hline \multirow[t]{2}{*}{ T status } & T0-T2 & 35 & 86.7 & \multirow{2}{*}{0.053} & & & \\
\hline & $\mathrm{T} 3$ and $\mathrm{T} 4$ & 41 & 58.9 & & & & \\
\hline \multirow[t]{2}{*}{ N status } & N0 & 43 & 86.7 & \multirow{2}{*}{0.110} & & & \\
\hline & N1 & 33 & 81.5 & & & & \\
\hline \multirow[t]{2}{*}{ R status } & R0 & 57 & 81.5 & \multirow{2}{*}{0.569} & & & \\
\hline & $\mathrm{R} 1$ & 19 & 28.2 & & & & \\
\hline \multirow[t]{2}{*}{ Adjuvant S-1 } & yes & 42 & NA & \multirow{2}{*}{$0.003 *$} & & & \\
\hline & no & 42 & 28.2 & & 4.370 & 1.989-10.298 & $<0.001 *$ \\
\hline
\end{tabular}

${ }^{*}$ Statistical significance $(p<0.050) .{ }^{\dagger} \log$ rank test. ${ }^{\ddagger}$ Cox proportional-hazards model. NA, not available; HR, hazard ratio; $95 \%$ CI, $95 \%$ confidence interval.

\subsection{Subgroup Analysis of the Prognostic Impact of S-1 Adjuvant Chemotherapy}

To evaluate the prognostic impact of adjuvant S-1 chemotherapy for patients with poor prognostic factors of perineural invasion, we compared the OS of the patients with perineural invasion between the S-1 group and the observation group. The profiles and tumour characteristics of the patients with perineural invasion are shown in Table S1. The median OS of the patients with perineural invasion in S-1 group was significantly better than that of the observation group (not available vs. 18.1 months, $p<0.001$ ) (Figure 3).

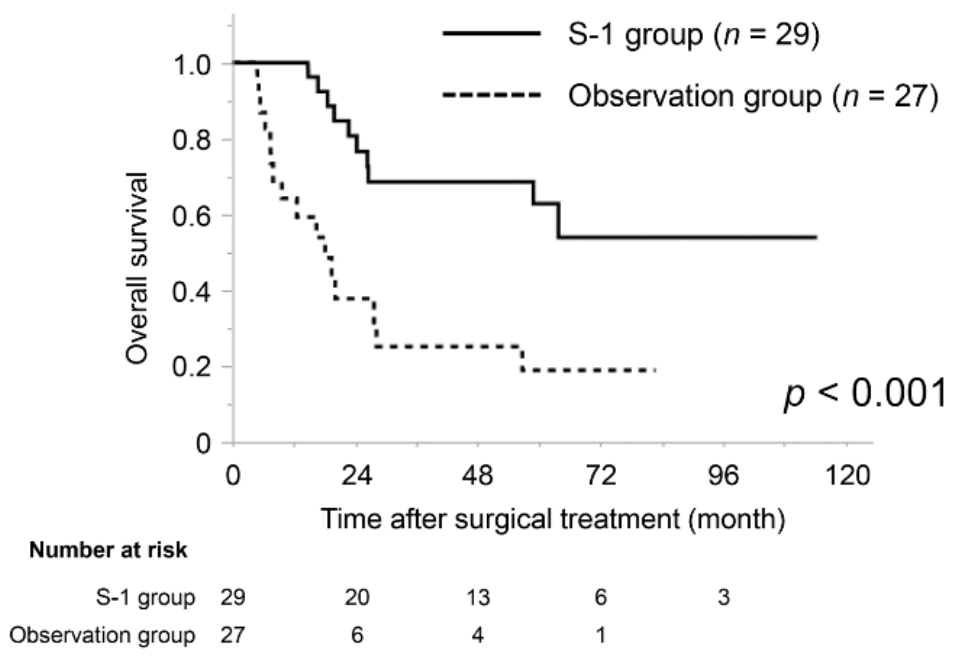

Figure 3. Kaplan-Meier curves for the overall survival of matched cohort patients with perineural invasion. The overall survival of the S-1 group was significantly better than that of the observation group $(p<0.001)$. 


\section{Discussion}

This study investigated the postoperative outcomes of BTC resection with the administration of S-1 as adjuvant chemotherapy. Given that patients considered to be at a high risk of recurrence would likely receive adjuvant chemotherapy, we performed a propensity score-matching analysis to reduce patient selection bias. In our matching cohort, both the RFS and OS of the patients in the S-1 group were significantly longer than those in the observation group. Furthermore, adjuvant S-1 chemotherapy might contribute to the improved prognosis of patients with perineural invasion.

Several studies have reported on adjuvant chemotherapy for BTC [10,11,15,18-26]. The multicentre randomized phase III trial PRODIGE 12-ACCORD 18 conducted by a French group failed to show the efficacy of gemcitabine and oxaliplatin (GEMOX) in treating BTC patients in an adjuvant setting [10]. Additionally, the randomized phase III trial BCAT from Japan also failed to show a significant efficacy of adjuvant gemcitabine chemotherapy [11]. The results of the present study differ from those reported by these two large randomized studies. One explanation may be related to the different adjuvant chemotherapeutic agents used, whereby S- 1 appears to achieve a better outcome when compared with gemcitabine in an adjuvant setting [14].

The efficacy of S-1 as adjuvant chemotherapy is well established for gastric cancer [4] and pancreatic cancer [9]. Regarding BTC, some studies showed that adjuvant S-1 improved the prognosis [14,15]. Given that S-1 contains the 5-fluorouracil (5-FU) prodrug tegafur [13], previous studies using 5-FU as adjuvant chemotherapy for BTC [21,23] have also suggested the potential efficacy of S-1. Recently, a multicentre randomized phase III trial of adjuvant chemotherapy for BTC (BILCAP) reported the efficacy of capecitabine, one of the prodrugs of 5-FU, with an OS of 53 months in the adjuvant group versus 36 months in the observation group ( $p=0.028$ ) [24]. It was reported that the allelic variants of CYP2A6, which is the metabolic enzyme of 5FU, were different between Caucasian and East Asian populations, but the pharmacokinetics of S-1 were not significantly different [27]. All of these previous studies support our current positive data for the use of S-1 in an adjuvant setting.

There are various reports on the prognostic factors after resection for BTC [28-30]. Perineural invasion $[31,32]$ was reported to be one of the poor prognostic factors. In our series, the patients with perineural invasion showed a poor prognosis and thus might benefit from adjuvant chemotherapy with S-1. Further studies are required to investigate the extent of benefit from adjuvant chemotherapy for BTC.

The present study has several limitations. The first was its retrospective nature. Although we analysed our data using propensity score matching, some selection bias may have remained. Second, our series contained a heterogeneous group of BTC patients and a small sample size. A future study with a homogeneous group of BTC patients and a larger sample size is required to confirm our results. Finally, the administration protocol of adjuvant S-1 was not unified, particularly the duration of administration. Further controlled prospective research is necessary, and the final results of the JCOG 1202 study [33], a randomized phase III trial of adjuvant S-1 therapy versus observation alone in resected BTC patients, are awaited.

In conclusion, we reported the efficacy of S- 1 as adjuvant chemotherapy after the resection of BTC using a propensity score matching analysis, and our results suggest that this approach might improve patients' prognoses, especially in patients with perineural invasion.

Supplementary Materials: The following are available online at https:/ / www.mdpi.com/2077-038 3/10/5/925/s1: Figure S1: Kaplan-Meier curves of recurrence-free survival and overall survival in the entire cohort, Table S1: Profiles and tumour characteristics of the patients with perineural invasion in each group of the matched cohort.

Author Contributions: Conceptualization, Y.M.; methodology, Y.M.; formal analysis, Y.M.; investigation, Y.M.; resources, R.K., A.N., R.N. (Rihito Nagata), T.M., Y.M., R.N. (Riki Ninomiya), M.K., A.M., N.K., and Y.B.; data curation, Y.M.; writing-original draft preparation, Y.M.; writing-review 
and editing, Y.B.; supervision, Y.B.; project administration, Y.M. and Y.B. All authors have read and agreed to the published version of the manuscript.

Funding: This research received no external funding.

Institutional Review Board Statement: The study was conducted according to the guidelines of the Declaration of Helsinki, and approved by the Institutional Review Board of Saitama Medical Centre, Saitama Medical University (approval no.: 2002; approval date: 1 November 2018) and Gyoda General Hospital (approval no.: 2019-1; approval date: 12 November 2019).

Informed Consent Statement: Informed consent was obtained from all the subjects involved in the study.

Data Availability Statement: The data presented in this study are available in the article.

Acknowledgments: We thank Hugh McGonigle and Melissa Crawford.

Conflicts of Interest: The authors declare no conflict of interest.

\section{References}

1. Bray, F.; Ferlay, J.; Soerjomataram, I.; Siegel, R.L.; Torre, L.A.; Jemal, A. Global cancer statistics 2018: GLOBOCAN estimates of incidence and mortality worldwide for 36 cancers in 185 countries. CA Cancer J. Clin. 2018, 68, 394-424. [CrossRef]

2. Bosman, F.T.; Carneiro, F.; Hruban, R.H.; Theise, N.D. WHO Classification of Tumours of the Digestive System, 4th ed.; IARC: Lyon, France, 2010.

3. Miyazaki, Y.; Kokudo, T.; Amikura, K.; Kageyama, Y.; Takahashi, A.; Ohkohchi, N.; Sakamoto, H. Survival of surgery for recurrent biliary tract cancer: A single-center experience and systematic review of literature. Jpn. J. Clin. Oncol. 2017, 47, 206-212. [CrossRef]

4. Sakuramoto, S.; Sasako, M.; Yamaguchi, T.; Kinoshita, T.; Fujii, M.; Nashimoto, A.; Furukawa, H.; Nakajima, T.; Ohashi, Y.; Imamura, H.; et al. Adjuvant chemotherapy for gastric cancer with S-1, an oral fluoropyrimidine. N. Engl. J. Med. 2007, 357, 1810-1820. [CrossRef]

5. $\quad$ Bang, Y.J.; Kim, Y.W.; Yang, H.K.; Chung, H.C.; Park, Y.K.; Lee, K.H.; Lee, K.W.; Kim, Y.H.; Noh, S.I.; Cho, J.Y.; et al. Adjuvant capecitabine and oxaliplatin for gastric cancer after D2 gastrectomy (CLASSIC): A phase 3 open-label, randomised controlled trial. Lancet 2012, 379, 315-321. [CrossRef]

6. Japanese Gastric Cancer Association. Japanese gastric cancer treatment guidelines 2014 (ver. 4). Gastric Cancer 2017, 20, 1-19. [CrossRef]

7. NIH Consensus Conference. Adjuvant therapy for patients with colon and rectal cancer. JAMA 1990, 264, 1444-1450. [CrossRef]

8. Hashiguchi, Y.; Muro, K.; Saito, Y.; Ito, Y.; Ajioka, Y.; Hamaguchi, T.; Hasegawa, K.; Hotta, K.; Ishida, H.; Ishiguro, M.; et al. Japanese Society for Cancer of the Colon and Rectum (JSCCR) guidelines 2019 for the treatment of colorectal cancer. Int. J. Clin. Oncol. 2020, 25, 1-42. [CrossRef]

9. Uesaka, K.; Boku, N.; Fukutomi, A.; Okamura, Y.; Konishi, M.; Matsumoto, I.; Kaneoka, Y.; Shimizu, Y.; Nakamori, S.; Sakamoto, H.; et al. Adjuvant chemotherapy of S-1 versus gemcitabine for resected pancreatic cancer: A phase 3, open-label, randomised, non-inferiority trial (JASPAC 01). Lancet 2016, 388, 248-257. [CrossRef]

10. Edeline, J.; Benabdelghani, M.; Bertaut, A.; Watelet, J.; Hammel, P.; Joly, J.P.; Boudjema, K.; Fartoux, L.; Bouhier-Leporrier, K.; Jouve, J.L.; et al. Gemcitabine and Oxaliplatin Chemotherapy or Surveillance in Resected Biliary Tract Cancer (PRODIGE 12-ACCORD 18-UNICANCER GI): A Randomized Phase III Study. J. Clin. Oncol. 2019, 37, 658-667. [CrossRef] [PubMed]

11. Ebata, T.; Hirano, S.; Konishi, M.; Uesaka, K.; Tsuchiya, Y.; Ohtsuka, M.; Kaneoka, Y.; Yamamoto, M.; Ambo, Y.; Shimizu, Y.; et al. Randomized clinical trial of adjuvant gemcitabine chemotherapy versus observation in resected bile duct cancer. Br. J. Surg. 2018, 105, 192-202. [CrossRef]

12. Shirasaka, T.; Shimamato, Y.; Ohshimo, H.; Yamaguchi, M.; Kato, T.; Yonekura, K.; Fukushima, M. Development of a novel form of an oral 5-fluorouracil derivative (S-1) directed to the potentiation of the tumor selective cytotoxicity of 5-fluorouracil by two biochemical modulators. Anticancer Drugs 1996, 7, 548-557. [CrossRef]

13. Shirasaka, T. Development history and concept of an oral anticancer agent S-1 (TS-1): Its clinical usefulness and future vistas. Jpn. J. Clin. Oncol. 2009, 39, 2-15. [CrossRef] [PubMed]

14. Kobayashi, S.; Terashima, T.; Shiba, S.; Yoshida, Y.; Yamada, I.; Iwadou, S.; Horiguchi, S.; Takahashi, H.; Suzuki, E.; Moriguchi, M.; et al. Multicenter retrospective analysis of systemic chemotherapy for unresectable combined hepatocellular and cholangiocarcinoma. Cancer Sci. 2018, 109, 2549-2557. [CrossRef]

15. Okabayashi, T.; Shima, Y.; Iwata, J.; Morita, S.; Sumiyoshi, T.; Sui, K.; Shimada, Y.; Iiyama, T. Characterization of Prognostic Factors and the Efficacy of Adjuvant S-1 Chemotherapy in Patients with Post-surgery Extrahepatic Bile Duct Cancer. Anticancer Res. 2017, 37, 7049-7056. [CrossRef]

16. Brierley, J.D.; Gospodarowicz, M.K.; Wittekind, C. International Union Against Cancer (UICC): TNM Classification of Malignant Tumors; Wiley: New York, NY, USA, 2017.

17. Dindo, D.; Demartines, N.; Clavien, P.A. Classification of surgical complications: A new proposal with evaluation in a cohort of 6336 patients and results of a survey. Ann. Surg. 2004, 240, 205-213. [CrossRef] 
18. Bergeat, D.; Turrini, O.; Courtin-Tanguy, L.; Truant, S.; Darnis, B.; Delpero, J.R.; Mabrut, J.Y.; Regenet, N.; Sulpice, L. Impact of adjuvant chemotherapy after pancreaticoduodenectomy for distal cholangiocarcinoma: A propensity score analysis from a French multicentric cohort. Langenbecks Arch. Surg. 2018, 403, 701-709. [CrossRef]

19. Murakami, Y.; Uemura, K.; Sudo, T.; Hayashidani, Y.; Hashimoto, Y.; Nakamura, H.; Nakashima, A.; Sueda, T. Adjuvant gemcitabine plus S-1 chemotherapy improves survival after aggressive surgical resection for advanced biliary carcinoma. Ann. Surg. 2009, 250, 950-956. [CrossRef] [PubMed]

20. Murakami, Y.; Uemura, K.; Sudo, T.; Hayashidani, Y.; Hashimoto, Y.; Nakamura, H.; Nakashima, A.; Sueda, T. Gemcitabine-based adjuvant chemotherapy improves survival after aggressive surgery for hilar cholangiocarcinoma. J. Gastrointest. Surg. 2009, 13, 1470-1479. [CrossRef]

21. Kim, Y.S.; Jeong, C.Y.; Song, H.N.; Kim, T.H.; Kim, H.J.; Lee, Y.J.; Hong, S.C. The efficacy of fluoropyrimidine-based adjuvant chemotherapy on biliary tract cancer after R0 resection. Chin. J. Cancer 2017, 36, 9. [CrossRef]

22. Yin, L.; Xu, Q.; Li, J.; Wei, Q.; Ying, J. The efficiency and regimen choice of adjuvant chemotherapy in biliary tract cancer: A STROBE-compliant retrospective cohort study. Medicine 2018, 97, e13570. [CrossRef] [PubMed]

23. Neoptolemos, J.P.; Moore, M.J.; Cox, T.F.; Valle, J.W.; Palmer, D.H.; McDonald, A.C.; Carter, R.; Tebbutt, N.C.; Dervenis, C.; Smith, D.; et al. Effect of adjuvant chemotherapy with fluorouracil plus folinic acid or gemcitabine vs observation on survival in patients with resected periampullary adenocarcinoma: The ESPAC-3 periampullary cancer randomized trial. JAMA 2012, 308, 147-156. [CrossRef] [PubMed]

24. Primrose, J.N.; Fox, R.P.; Palmer, D.H.; Malik, H.Z.; Prasad, R.; Mirza, D.; Anthony, A.; Corrie, P.; Falk, S.; Finch-Jones, M.; et al. Capecitabine compared with observation in resected biliary tract cancer (BILCAP): A randomised, controlled, multicentre, phase 3 study. Lancet Oncol. 2019, 20, 663-673. [CrossRef]

25. Nassour, I.; Mokdad, A.A.; Porembka, M.R.; Choti, M.A.; Polanco, P.M.; Mansour, J.C.; Minter, R.M.; Wang, S.C.; Yopp, A.C. Adjuvant Therapy Is Associated with Improved Survival in Resected Perihilar Cholangiocarcinoma: A Propensity Matched Study. Ann. Surg. Oncol. 2018, 25, 1193-1201. [CrossRef] [PubMed]

26. Kemp Bohan, P.M.; Kirby, D.T.; Chick, R.C.; Bader, J.O.; Clifton, G.T.; Vreeland, T.J.; Nelson, D.W. Adjuvant Chemotherapy in Resectable Gallbladder Cancer is Underutilized Despite Benefits in Node-Positive Patients. Ann. Surg. Oncol. 2020. [CrossRef]

27. Chuah, B.; Goh, B.C.; Lee, S.C.; Soong, R.; Lau, F.; Mulay, M.; Dinolfo, M.; Lim, S.E.; Soo, R.; Furuie, T.; et al. Comparison of the pharmacokinetics and pharmacodynamics of S-1 between Caucasian and East Asian patients. Cancer Sci. 2011, 102, 478-483. [CrossRef]

28. Matsukuma, S.; Tokumitsu, Y.; Shindo, Y.; Matsui, H.; Nagano, H. Essential updates to the surgical treatment of biliary tract cancer. Ann. Gastroenterol. Surg. 2019, 3, 378-389. [CrossRef]

29. Beetz, O.; Klein, M.; Schrem, H.; Gwiasda, J.; Vondran, F.W.R.; Oldhafer, F.; Cammann, S.; Klempnauer, J.; Oldhafer, K.J.; Kleine, M. Relevant prognostic factors influencing outcome of patients after surgical resection of distal cholangiocarcinoma. BMC Surg. 2018, 18, 56. [CrossRef] [PubMed]

30. Petrova, E.; Ruckert, F.; Zach, S.; Shen, Y.; Weitz, J.; Grutzmann, R.; Wittel, U.A.; Makowiec, F.; Hopt, U.T.; Bronsert, P.; et al. Survival outcome and prognostic factors after pancreatoduodenectomy for distal bile duct carcinoma: A retrospective multicenter study. Langenbecks Arch. Surg. 2017, 402, 831-840. [CrossRef]

31. Bhuiya, M.R.; Nimura, Y.; Kamiya, J.; Kondo, S.; Fukata, S.; Hayakawa, N.; Shionoya, S. Clinicopathologic studies on perineural invasion of bile duct carcinoma. Ann. Surg. 1992, 215, 344-349. [CrossRef] [PubMed]

32. Yamaguchi, R.; Nagino, M.; Oda, K.; Kamiya, J.; Uesaka, K.; Nimura, Y. Perineural invasion has a negative impact on survival of patients with gallbladder carcinoma. Br. J. Surg. 2002, 89, 1130-1136. [CrossRef]

33. Nakachi, K.; Konishi, M.; Ikeda, M.; Mizusawa, J.; Eba, J.; Okusaka, T.; Ishii, H.; Fukuda, H.; Furuse, J. A randomized Phase III trial of adjuvant S-1 therapy vs. observation alone in resected biliary tract cancer: Japan Clinical Oncology Group Study (JCOG1202, ASCOT). Jpn. J. Clin. Oncol. 2018, 48, 392-395. [CrossRef] [PubMed] 